\title{
Electron hybrid code simulation of whistler-mode chorus generation with real parameters in the Earth's inner magnetosphere
}

\author{
Yuto Katoh ${ }^{1 *}$ (D) and Yoshiharu Omura ${ }^{2}$ (D)
}

\begin{abstract}
We carry out a self-consistent simulation of the generation process of whistler-mode chorus by a spatially one-dimensional electron hybrid code, by assuming the magnetic field inhomogeneity corresponding to $L=4$ of the dipole field. Chorus emissions with rising tones are reproduced in the simulation result, while the frequency range, sweep rate, and the amplitude profiles in the spectra of the reproduced elements are consistently explained by the nonlinear wave growth theory. We compare the simulation results with the observation by the Cluster spacecraft (Santolik et al. in J Geophys Res 108:1278, 2003, doi:10.1029/2002JA009791; Santolik in Nonlinear Process Geophys 15:621-630, 2008) and reveal similarities of the spectral fine structure of reproduced chorus elements with the observation. On the other hand, there is no gap at half the gyrofrequency in the spectra of the reproduced chorus elements, which is evident in the observation. This difference implies that the mechanism of a gap at half the gyrofrequency is governed by the process that is not described by the spatially one-dimensional simulation treating purely parallel propagating electromagnetic waves.
\end{abstract}

Keywords: Magnetosphere, Whistler-mode chorus, Numerical experiments

\section{Introduction}

Whistler-mode chorus emissions are electromagnetic plasma waves observed in the Earth's magnetosphere. Chorus emissions are a group of coherent wave elements whose frequency varies in time (Santolik et al. 2003, 2014). The typical frequency range of chorus is from 0.2 to $0.8 \Omega_{\mathrm{e} 0}$, where $\Omega_{\mathrm{e} 0}$ is the electron gyrofrequency at the magnetic equator. Chorus are often classified into the lower band $\left(0.2-0.5 \Omega_{\mathrm{e} 0}\right)$ and the upper band $(0.5-$ $0.8 \Omega_{\mathrm{e} 0}$ ) because of the different propagation properties and characteristics appeared in the spectra accompanied with a distinct gap at half the gyrofrequency (e.g., Bell et al. 2009). Since whistler-mode waves satisfy the cyclotron resonance condition with electrons in the wide range of kinetic energy and pitch angle, resonant scattering of energetic electrons in the magnetosphere has been

\footnotetext{
*Correspondence: yuto@stpp.gp.tohoku.ac.jp

1 Department of Geophysics, Graduate School of Science, Tohoku University, 6-3 Aramaki-aza-aoba, Aoba, Sendai, Miyagi 980-8578, Japan Full list of author information is available at the end of the article
}

extensively discussed by various approaches: by a diffusion code based on the quasi-linear theory (e.g., Thorne et al. 2010), by a test-particle analysis (e.g., Albert 2001; Bortnik et al. 2008), and by an analytic approach (e.g., Lakhina et al. 2010). On the other hand, wave-particle interactions between chorus and energetic electrons including the wave excitation process can only be investigated by a self-consistent simulation code, as we use in the present study.

Self-consistent particle simulations reproduced the generation process of chorus with rising tones and revealed nonlinear properties of the chorus generation (e.g., Katoh and Omura 2007, 2011, 2013). Chorus are generated at the magnetic equator, where the intensity of the background magnetic field becomes minimum along a field line, and propagate away from the equator with increasing their wave amplitude. Simulation results clarified that the magnetic field inhomogeneity along a field line controls the threshold of the wave amplitude required for the chorus generation (Katoh and Omura 2013) and the frequency sweep rate of chorus changes 
depending on the wave amplitude of each chorus element (Katoh and Omura 2011). These properties of chorus have been clearly explained by the nonlinear wave growth theory proposed for the generation mechanism of chorus (Omura et al. 2008, 2009, 2012).

Previous studies clarified that the magnetic field inhomogeneity along a field line is an important factor controlling the spectral properties of chorus. On the other hand, the spatial scale of the simulation system used in previous particle simulations is smaller than that of the real magnetosphere, and therefore larger magnetic field inhomogeneities have been used (Katoh and Omura 2007, 2011, 2013; Hikishima et al. 2009), because of the limitation of computational resources. The difference of the spatial scale between the simulation system and the real magnetosphere prevents us to compare the simulation results with in situ observation of chorus by satellites. In the present study, we have overcome this difficulty with the help of sufficient computational resources provided by the High-Performance Computing Infrastructure projects and collaborations with supercomputer centers of universities in Japan and have carried out electron hybrid simulations in the simulation system corresponding to the real magnetosphere. We present simulation results for a magnetic field inhomogeneity corresponding to $L=4$ of the dipole field and compare the results with spectral properties of chorus observed in the magnetosphere.

\section{Simulation model and initial condition}

We use an originally developed electron hybrid code (e.g., Katoh and Omura 2004, 2006; Katoh et al. 2005a, b), which treats the background cold electrons as a fluid and energetic electrons as particles by the standard particlein-cell method with fully relativistic effects. This code has reproduced the generation process of chorus with rising tones (e.g., Katoh and Omura 2007, 2011, 2013; Omura et al. 2008). We assume a spatially one-dimensional simulation system along a field line. Katoh and Omura (2013) revealed that the magnetic field inhomogeneity is an important factor controlling the threshold amplitude for the chorus generation. In the present study, we use the magnetic field inhomogeneity along a dipole field line of $L=4$. The number of grid points is 65,536 and the spatial scale of the simulation system is $6553.6 \mathrm{c} \Omega_{\mathrm{e} 0}{ }^{-1}$, where $\mathrm{c}$ is the speed of light, corresponding to the spatial extent of $18,500 \mathrm{~km}$ from the magnetic equator in both hemispheres. We assume that the plasma frequency $\omega_{\text {pe }}$ of cold electrons at the magnetic equator is $2.4 \Omega_{\mathrm{e} 0}$ and that the cold plasma density is constant in space and time in the simulation system. We load energetic electrons in the simulation system taking into account the adiabatic motion of electrons along a field line. The magnetic field line is filled with energetic electrons as the initial condition of simulations. We use one billion of particles representing energetic electrons in the simulation system. We assume the initial velocity distribution of energetic electrons is anisotropic (Fig. 1a), which drives an instability generating a band of whistler-mode waves. We use the following equations for the initial distribution function of energetic electrons in the momentum space.

$$
f\left(u_{\|}, u_{\perp}\right)=C \exp \left(-\frac{u_{\|}^{2}}{2 U_{t \|}^{2}}\right) g\left(u_{\perp}\right)
$$

and

$g\left(u_{\perp}\right)=\frac{1}{1-\beta}\left\{\exp \left(-\frac{u_{\perp}^{2}}{2 U_{t \perp}^{2}}\right)-\exp \left(-\frac{u_{\perp}^{2}}{2 \beta U_{t \perp}^{2}}\right)\right\}$,

where $u_{\|}$and $u_{\perp}$ are momenta parallel and perpendicular to the background magnetic field, respectively, $U_{t \|}$ and $U_{t \perp}$ are thermal momenta parallel and perpendicular to the background magnetic field, respectively, $\beta$ is a parameter describing the depth of the loss cone distribution, and we use $\beta=0.5$. We assume that $U_{t||} / c$ and $U_{t \perp} / c$ are 0.2705 and 0.46852 , respectively. Here, the temperature anisotropy is computed by $A_{T}=(1+\beta)$ $U_{t \perp}^{2} / U_{t \mid l}^{2}-1$ (cf. Tang et al. 2014) and is 3.5 for the assumed thermal momenta. Figure $1 \mathrm{~b}$ represents the energy spectrum of electrons used in the present study. We assume the number density of energetic electrons $N_{\mathrm{h}}$ at the equator is $9.9 \times 10^{-4} N_{0}$, where $N_{0}$ is the number density of cold electrons at the magnetic equator of the simulation system. The initial parameters used in the simulation are summarized in Table 1.

\section{Results and discussion}

We conducted the simulation up to $40,000 \Omega_{\mathrm{e} 0}^{-1}$, corresponding to $0.8 \mathrm{~s}$ for the background magnetic field intensity of 285 nT (cf. Santolik et al. 2003; hereafter we use this parameter for the conversion of simulation results to real values). The computational time used in this simulation is almost 1 week with 1024 cores.

In the simulation result, a band of whistler-mode waves are excited through the instability driven by the temperature anisotropy of energetic electrons, and the amplitude of whistler-mode waves grows in time and becomes coherent. When the amplitude exceeds a certain threshold level, the contribution of the wave magnetic field $B_{\mathrm{w}}$ on the Lorentz force acting on energetic electrons becomes non-negligible, and then the balance between the nonlinear Lorentz force $q_{\mathrm{e}} v \times B_{\mathrm{w}}$ and the mirror force due to the background magnetic field inhomogeneity results in convective nonlinear wave growth 


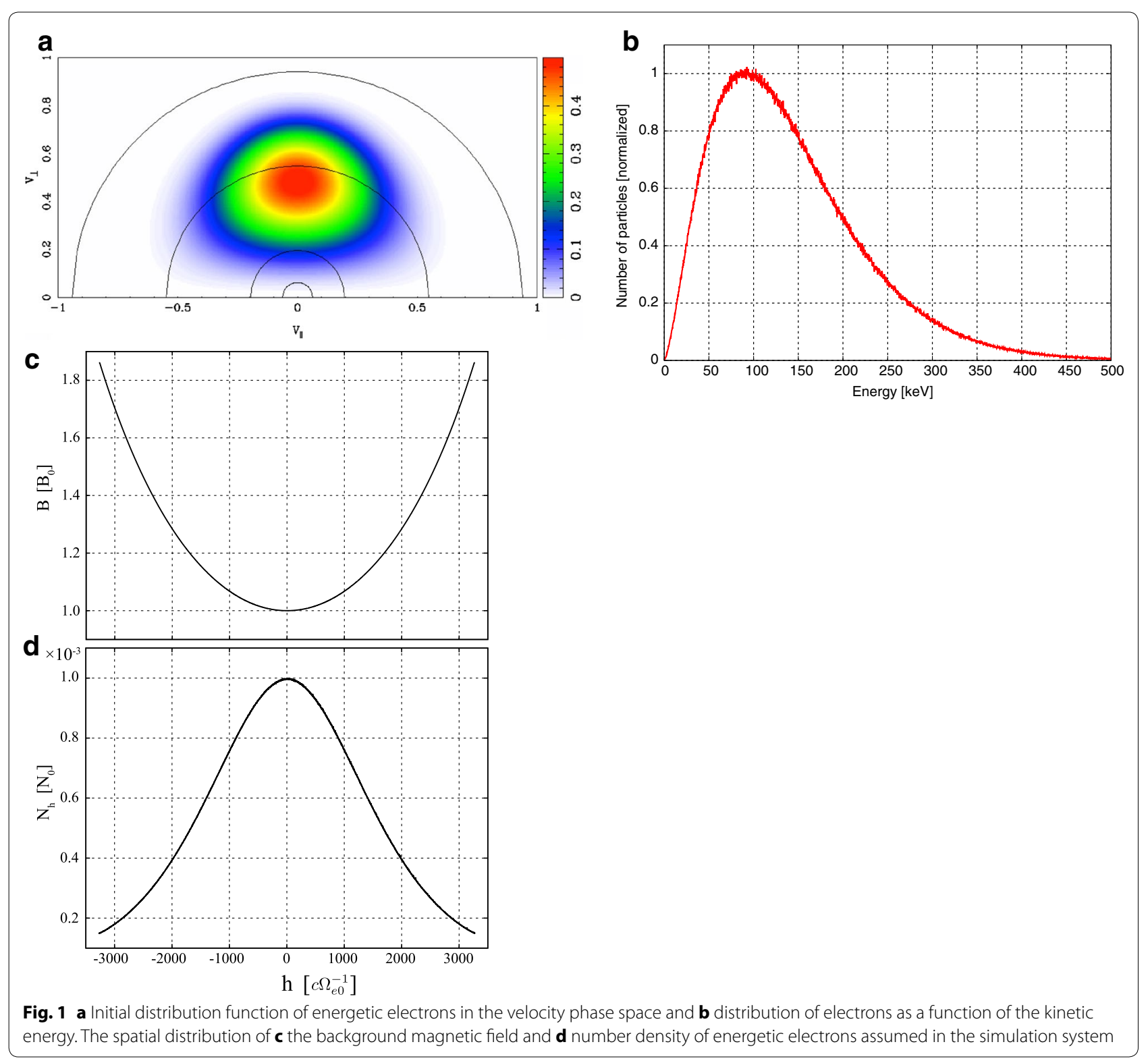

due to the formation of an asymmetric electromagnetic electron hole in the velocity phase space, where $q_{\mathrm{e}}$ is the charge of an electron. Although the background magnetic field inhomogeneity is zero at the magnetic equator, the electron hole can be deformed to be asymmetric with respect to the wave magnetic field, because a term related to the second derivative of the wave phase (namely the frequency variation in time) plays the same role as the mirror force on the dynamics of resonant particles. The presence of the asymmetric hole in the velocity phase space results in the formation of a net resonant current parallel to the wave electric field. The intensity of resonant current maximized for a wave component having a specific wave phase variation. Since the resonant current contributes the amplification of the wave amplitude, coherent wave elements whose wave phase variation satisfies the condition of the maximum resonant current undergo significant amplification due to the selective nonlinear growth. In the wave growth process, the other resonant current parallel to the wave magnetic field is also formed due to the electron hole, and it contributes to the frequency shift to higher frequencies. Coherent wave elements with rising tones therefore emerge from a band of whistler-mode waves in the region close to the magnetic equator. The coherent wave elements propagate away from the equator with growing wave amplitudes, 
Table 1 Summary of the initial parameters used in the simulation

\begin{tabular}{lc}
\hline Plasma frequency $\omega_{\text {pe }}$ of the cold electrons & $2.4 \Omega_{\mathrm{e} 0}$ \\
Time step $\Delta t$ & $0.05 \Omega_{\mathrm{e} 0}^{-1}$ \\
Grid spacing $\Delta x$ & $0.1 \mathrm{c} \Omega_{\mathrm{e} 0}^{-1}$ \\
Number of grid points & 65,536 \\
Number of particles & $1,342,177,280$ \\
Density ratio between cold $\left(N_{0}\right)$ and energetic electrons $\left(N_{\mathrm{h}}\right)$ at the magnetic equator & $9.9 \times 10^{-4}$ \\
Thermal momenta of energetic electrons parallel and perpendicular to the background magnetic field $\left(U_{t \|}\right.$ and $\left.U_{t \perp}\right)$ & $U_{t \mid \|} / \mathrm{C}=0.2705$ \\
& $U_{t \perp} / \mathrm{C}=0.46852$ \\
Temperature anisotropy of energetic electrons $\left(A_{T}\right) ; A_{T}=(1+\beta) U_{t \perp}^{2} / U_{t \|}^{2}-1$ & 3.5 and $\beta=0.5$ \\
\hline
\end{tabular}

while the timescale of the wave growth of the coherent elements is much faster than that of the band of whistler-mode waves appeared in the elementary stage of the simulation. Figure 2a shows the spatial distribution of the spectra measured at the final stage of the simulation, indicating that multiple coherent wave elements are generated from the magnetic equator $(h=0)$ and propagate away from the equator toward both hemispheres. Because we used random numbers to place particles in the simulation system, the spatial distribution of energetic electrons is not perfectly symmetric and therefore waves generated in the simulation system are not exactly the same in the Northern and Southern Hemispheres. The spatial distribution of the spectra clearly indicates that the wave amplitude of coherent elements increases during the propagation away from the equator and clarifies that the rising tone of each element is already formed at the equator. These results are consistent with our previous simulation studies (Katoh and Omura 2007, 2011, 2013).

Figure $2 \mathrm{~b}$ indicates the wave magnetic field spectra observed at $h=-655.36 \mathrm{c} \Omega_{\mathrm{e} 0}^{-1}$. Two distinct chorus elements with rising tones are identified in Fig. 2b. The wave magnetic field amplitude of each element reaches up to $0.1 \%$ of the background magnetic field intensity. The wave frequency changes from 0.26 to $0.55 \Omega_{\mathrm{e} 0}$ during the time interval of $30,000 \Omega_{\mathrm{e} 0}^{-1}$, and therefore, the frequency sweep rate is roughly estimated to be $3.89 \mathrm{kHz} / \mathrm{s}$, which is in agreement with the typical frequency sweep rates of rising tone chorus observed in the magnetosphere (e.g., Santolik 2008). When we focus on the fine-scale spectral structure of reproduced elements, we find that the frequency sweep rate of each element is not constant but varies in time. Here, we define the chorus element appearing in the relatively lower frequency range as "Element 1" and another in the higher frequency range as "Element 2." The spectral structure of Element 1 indicates that the sweep rate is small in the beginning of the frequency variation (corresponds to the leading edge of Element 1 at $h=-655.36 \mathrm{c} \Omega_{\mathrm{e} 0}^{-1}$ during the time interval from 10,000 to $20,000 \Omega_{\mathrm{e} 0}^{-1}$ ), and then the sweep rate increases with increasing the wave amplitude (from 20,000 to $28,000 \Omega_{\mathrm{e} 0}^{-1}$ ), and eventually the sweep rate becomes small again for the trailing edge of
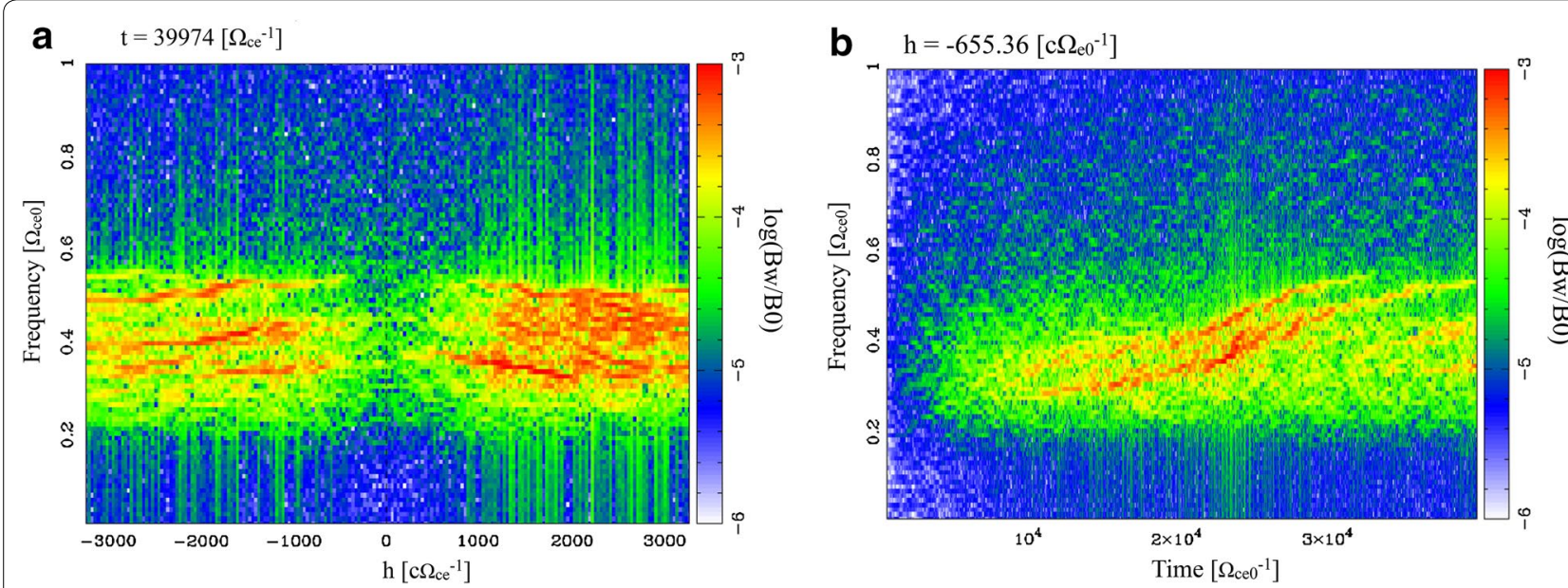

Fig. 2 a The spatial distribution of wave magnetic field spectra observed in the simulation system at the final stage of the simulation and $\mathbf{b}$ the spectra observed at $h=-655.36 \mathrm{c} \Omega_{\mathrm{e} 0}^{-1}$ 
Element 1. The similar spectral fine structure is also identified for Element 2. The temporal variation of the frequency sweep rate identified in the simulation result can be explained by the relation between the sweep rate and the wave amplitude derived from the nonlinear wave growth theory (Omura et al. 2008, 2009). Figure 3a indicates the maximum amplitude of each frequency bin corresponding to each element at $h=0$. We note that the wave amplitude of elements shown in Fig. 3a is one order of the magnitude smaller than that shown in Fig. $2 b$ (at $h=-655.36 \mathrm{c} \Omega_{\mathrm{e} 0}^{-1}$ ) because of the nonlinear wave growth during the propagation away from the equator. We compare the amplitude profiles of the reproduced elements with estimations of the threshold (Omura et al. 2009) and optimum amplitude (Omura and Nunn 2011), proposed based on the nonlinear wave growth theory. Here, we discuss the depth of the electron hole (hereafter we describe "Q"), which is an important parameter related to the threshold and optimum amplitudes; the threshold amplitude is inversely proportional to the square of $Q$ and the optimum amplitude is linearly proportional to $Q$. The distribution of particles in the velocity phase space around the electron hole significantly varies depending on the evolution of wave electromagnetic fields, and the variation of the flux of resonant electrons, which are mostly untrapped by coherent wave elements and move around the electron hole, contributes to the variation of $Q$ by increasing the number of resonant particles outside of the electron hole. While it is difficult to exactly determine $Q$, based on the simulation results and estimations used in our previous studies, we assumed $Q=0.5$ in the present study as a typical value of the depth of the hole. We find in Fig. 3a that both of the reproduced elements emerge at the frequency where the wave amplitude exceeds the threshold and that the reproduced frequency profiles follow those of the optimum amplitude. In addition, we estimated sweep rates of chorus elements by Omura et al. (2008)

$$
\frac{\partial \omega}{\partial t}=\frac{0.4 \chi}{\gamma \xi} \frac{V_{\perp 0}}{c} \frac{\omega}{\Omega_{\mathrm{e} 0}}\left(1-\frac{V_{\mathrm{R}}}{V_{\mathrm{g}}}\right)^{-2} \frac{B_{\mathrm{W}}}{B_{0}} \Omega_{\mathrm{e} 0}^{2}
$$

where $\chi^{2}=\left(1+\xi^{2}\right)^{-1}, \xi^{2}=\omega\left(\Omega_{\mathrm{e} 0}-\omega\right) / \omega_{\mathrm{pe}}^{2}, \gamma$ is the Lorentz factor, $V_{\perp 0}$ is an average value of $v_{\perp}$ of the initial velocity distribution of energetic electrons at the magnetic equator, $V_{\mathrm{R}}$ is the resonance velocity, and $V_{\mathrm{g}}$ is the group velocity. Figure $3 \mathrm{~b}$ shows the wave magnetic field spectra of southward propagating waves observed at $h=0$. We superposed sweep rates estimated every $2000 \Omega_{\mathrm{e} 0}^{-1}$ for both elements, while we extracted instantaneous amplitudes and frequencies of each element from the waveform of southward propagating waves observed at $h=0$ and used them in the estimation of the sweep rates. We find that the estimated sweep rates basically match the frequency variation of each element appeared in the spectra. Figure 3 reveals that the chorus generation process reproduced in the simulation is consistently explained by the nonlinear wave growth theory.

Next, we compare the simulation results with observed chorus elements in the magnetosphere. For the comparison, we refer detailed analysis made by Santolik et al. (2003) and Santolik (2008) for the fine structure of chorus
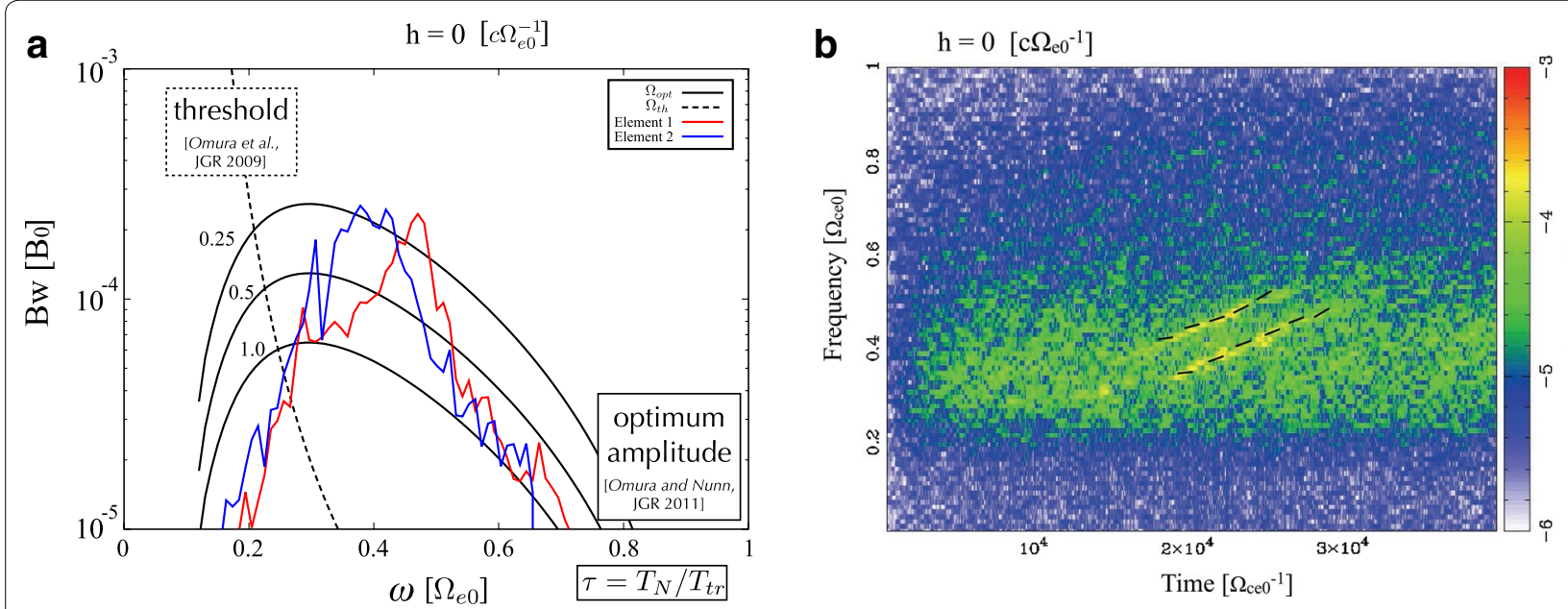

Fig. 3 a The frequency profiles of the maximum wave magnetic field amplitude of each element observed at the magnetic equator, $h=0$. The estimated threshold and optimum amplitude are also shown, where $\mathrm{T}$ is the ratio of the nonlinear transition time $T_{\mathrm{N}}$ to the nonlinear trapping period $T_{\text {tr }}$ (cf. Omura and Nunn 2011). b The wave magnetic field spectra of southward propagating waves observed at $h=0$. Solid lines superposed on the spectra indicate estimated frequency sweep rates based on the nonlinear wave growth theory 
observed by the Cluster spacecraft during a geomagnetically disturbed period on April 18, 2002. The observation results are briefly summarized as follows.

The location of the Cluster spacecraft during the observed event was in the nightside of the magnetosphere (2100 MLT) at a radial distance of 4.4 Earth's radii close to the magnetic equatorial plane. Chorus elements with rising tones were detected in the frequency range less than half the local electron gyrofrequency, corresponding to the frequency range of the lower band chorus. They analyzed the waveform of the chorus elements and showed that the instantaneous wave electric field amplitude ranged typically between a few $\mathrm{mV} / \mathrm{m}$ and about $10 \mathrm{mV} / \mathrm{m}$ but sometimes reaches more than $30 \mathrm{mV} / \mathrm{m}$. Santolik (2008) presented results of further analysis of this event and showed that the typical wave magnetic field amplitude is a few hundreds of $\mathrm{pT}$, corresponding to $0.1 \%$ of the background magnetic field intensity.

In Fig. 4, we compare the spectra of the chorus elements reproduced in the simulation result with those of chorus elements measured by the Cluster spacecraft. The left panel of Fig. 4 is the same result as shown in Fig. $2 \mathrm{~b}$ except for the scale of the horizontal and vertical axes adjusted to the observation. Figure 4 indicates similarities of the spectral fine structure of reproduced chorus elements in the simulation with the observation. We find that Element 1 appears in the spectra from $0.27 \Omega_{\mathrm{e} 0}^{-1}$, which is the same frequency of the observed element. The sweep rate of Element 1 changes as a function of the wave frequency as we discussed above, while the sweep rate of the observed chorus element shows the similar variation up to $0.5 \Omega_{\mathrm{e} 0}^{-1}$. These results of the qualitative comparison indicate similarities between the simulation

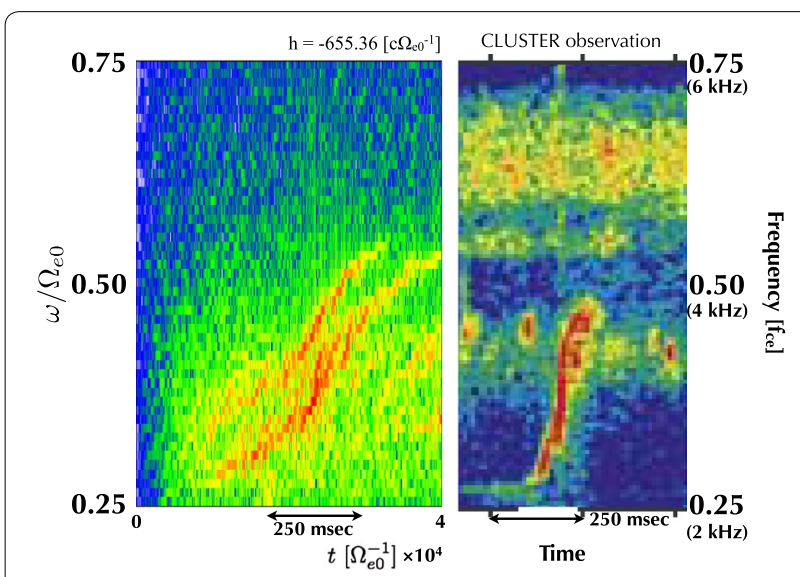

Fig. 4 Comparison of the spectra of the chorus elements reproduced in the simulation result with a chorus element observed by the Cluster spacecraft (Santolik et al. 2003) results and the observation. On the other hand, we did not find a gap at half the gyrofrequency in both Elements 1 and 2, which is evident in the observation. This difference implies that the mechanism of a gap at $0.5 \Omega_{\mathrm{e} 0}$ is governed by the physical process that is not described by the spatially one-dimensional system treating purely parallel propagating electromagnetic waves, such as the nonlinear damping through Landau resonance (Omura et al. 2009; Yagitani et al. 2014) and/or properties of obliquely propagating whistler-mode waves (e.g., Bell et al. 2009; Katoh 2014; Nunn and Omura 2015).

Macúšová et al. (2010) analyzed 13 events of whistlermode chorus observed by the Cluster spacecraft during different levels of geomagnetic activity and showed that the frequency sweep rate decreases with the decreasing background plasma density. Based on the nonlinear wave growth theory, Omura et al. (2015) showed that the frequency sweep rate decreases with increasing $\omega_{\mathrm{pe}} / \Omega_{\mathrm{e} 0}$ in the frequency range higher than $0.1 \Omega_{\mathrm{e} 0}$. Therefore, the relationship between the sweep rate and plasma density reported in Macúšová et al. can be consistently explained by the nonlinear wave growth theory. In addition, Katoh and Omura (2011) showed by a series of electron hybrid simulations that the frequency sweep rate of chorus elements increases with increasing $N_{\mathrm{h}}$. Since properties of the chorus generation are controlled by various factors, properties revealed by theory and simulation results should be examined further by comparison with observations in the future study.

\section{Summary}

In the present study, we carried out a self-consistent simulation of the generation process of whistler-mode chorus by the spatially one-dimensional electron hybrid code. We assumed the magnetic field inhomogeneity corresponding to $L=4$ of the dipole field in the simulation system. In the simulation result, chorus emissions with rising tones are reproduced, while the frequency range, sweep rate, and the amplitude profiles of the reproduced elements are consistently explained by the nonlinear wave growth theory. We compared the reproduced spectra of chorus with the observation result by the Cluster spacecraft (Santolik et al. 2003; Santolik 2008) and found similarities between them, except for a gap at half the gyrofrequency. While we focused on the spectral properties of chorus generated under the realistic initial condition of the background magnetic field, the dynamics of resonant electrons under the realistic conditions should be investigated in detail and is left as our future study.

Recent progress of supercomputer facilities enables us to reproduce the chorus generation process in the realistic magnetospheric configurations. Simulation studies of interactions between chorus and energetic 
electrons under the realistic plasma environment will provide important clues in understanding the observation. As a part of the international collaboration of the exploration of wave-particle interactions occurring in the inner magnetosphere, the upcoming JAXA satellite ERG (Exploration of energization and Radiation in Geospace) (Miyoshi et al. 2012) will provide valuable datasets for the investigation of nonlinear waveparticle interactions in the inner magnetosphere. In particular, Software-type Wave-Particle Interaction Analyzer (SWPIA) (Katoh et al. 2013; Hikishima et al. 2014) on board the ERG satellite will provide instantaneous wave and particle data measured in the chorus generation region with the extremely high time resolution less than the electron gyroperiod, which is a unique dataset for the investigation of nonlinear wave-particle interactions. For the thorough understanding of the chorus generation process and the energization process of relativistic electrons, simulations with the plasma environment and velocity distributions observed in the real magnetosphere are important in the future study.

\section{Authors' contributions}

YK carried out numerical experiments and analyzed the simulation results. YO was involved in the discussion of the simulation results. Both authors read and approved the final manuscript.

\section{Author details}

1 Department of Geophysics, Graduate School of Science, Tohoku University, 6-3 Aramaki-aza-aoba, Aoba, Sendai, Miyagi 980-8578, Japan. ${ }^{2}$ Research Institute for Sustainable Humanosphere, Kyoto University, Gokasho, Uji, Kyoto 611-0011, Japan.

\section{Acknowledgements \\ The computer simulation was performed on the KDK computer system at the Research Institute for Sustainable Humanosphere, Kyoto University, and the computational resources of the $\mathrm{HPCl}$ system provided by the Research Institute for Information Technology, Kyushu University; the Information Technology Center, Nagoya University; and the Cyberscience Center, Tohoku University, through the HPCI System Research Project (Project ID: hp160131). This study is supported by Grants-in-Aid for Scientific Research (26287120, $15 \mathrm{H} 05747,15 \mathrm{H} 05815$, and $15 \mathrm{H} 03730$ ) of Japan Society for the Promotion of Science. This research is also supported by "Advanced Computational Scientific Program" of Research Institute for Information Technology, Kyushu Univer- sity, and by "Computational Joint Research Program (Collaborative Research Project on Computer Science with High-Performance Computing)" at the Institute for Space-Earth Environmental Research, Nagoya University.}

\section{Competing interests}

The authors declare that they have no competing interests.

Received: 28 September 2016 Accepted: 16 November 2016 Published online: 25 November 2016

\section{References}

Albert JM (2001) Comparison of pitch angle diffusion by turbulent and monochromatic whistler waves. J Geophys Res 106(A5):8477. doi:10.102 9/2000JA000304

Bell TF, Inan US, Haque N, Pickett JS (2009) Source regions of banded chorus. Geophys Res Lett 36:L11101. doi:10.1029/2009GL037629
Bortnik J, Thorne RM, Inan US (2008) Nonlinear interaction of energetic electrons with large amplitude chorus. Geophys Res Lett 35:L21102. doi:10.10 29/2008GL035500

Hikishima M, Yagitani S, Omura Y, Nagano I (2009) Full particle simulation of whistler-mode rising chorus emissions in the magnetosphere. J Geophys Res 114:A01203. doi:10.1029/2008JA013625

Hikishima M, Katoh Y, Kojima H (2014) Evaluation of waveform data processing in wave-particle interaction analyzer. Earth Planets Space 66:63. doi:10.1186/1880-5981-66-63

Katoh Y (2014) A simulation study of the propagation of whistler-mode chorus in the Earth's inner magnetosphere. Earth Planets Space 66:6. doi:10.1186/1880-5981-66-6

Katoh Y, Omura Y (2004) Acceleration of relativistic electrons due to resonant scattering by whistler mode waves generated by temperature anisotropy in the inner magnetosphere. J Geophys Res 109:A12214. doi:10.1029/20 04JA010654

Katoh Y, Omura Y (2006) A study of generation mechanism of vlf triggered emission by self-consistent particle code. J Geophys Res 111:A12207. doi: 10.1029/2006JA011704

Katoh Y, Omura Y (2007) Computer simulation of chorus wave generation in the Earth's inner magnetosphere. Geophys Res Lett 34:L03102. doi:10.10 29/2006GL028594

Katoh Y, Omura Y (2011) Amplitude dependence of frequency sweep rates of whistler mode chorus emissions. J Geophys Res 116:A07201. doi:10.1029 /2011JA016496

Katoh Y, Omura Y (2013) Effect of the background magnetic field inhomogeneity on generation processes of whistler-mode chorus and broadband hiss-like emissions. J Geophys Res Space Phys 118:4189-4198 doi:10.1002/jgra.50395

Katoh Y, Ono T, lizima M (2005a) A numerical study on the resonant scattering process of relativistic electrons via whistler-mode waves in the outer radiation belt. In: Pulkkinen T, Friedel RHW, Tsyganenko N (eds) The inner magnetosphere: physics and modeling, Geophys. Monogr. Ser., vol 155. AGU, Washington, DC, pp 33-39

Katoh Y, Ono T, lizima M (2005b) Numerical simulation of resonant scattering of energetic electrons in the outer radiation belt. Earth Planets Space 57(2):117-124. doi:10.1186/BF03352555

Katoh Y, Kitahara M, Kojima H, Omura Y, Kasahara S, Hirahara M, Miyoshi Y, Seki K, Asamura K, Takashima T, Ono T (2013) Significance of wave-particle interaction analyzer for direct measurements of nonlinear wave-particle interactions. Ann Geophys 31:503-512. doi:10.5194/angeo-31-503-2013

Lakhina GS, Tsurutani BT, Verkhoglyadova OP, Pickett JS (2010) Pitch angle transport of electrons due to cyclotron interactions with the coherent chorus subelements. J Geophys Res 115:A00F15. doi:10.1029/2009JA014885

Macúšová E et al (2010) Observations of the relationship between frequency sweep rates of chorus wave packets and plasma density. J Geophys Res 115:A12257. doi:10.1029/2010JA015468

Miyoshi Y, Ono T, Takashima T, Asamura K, Hirahara M, Kasaba Y, Matsuoka A, Kojima H, Shiokawa K, Seki K, Fujimoto M, Nagatsuma T, Cheng CZ, Kazama Y, Kasahara S, Mitani T, Matsumoto H, Higashio N, Kumamoto A, Yagitani S, Kasahara Y, Ishisaka K, Blomberg L, Fujimoto A, Katoh Y, Ebihara Y, Omura Y, Nose M, Hori T, Miyashita Y et al (2012) The energization and radiation in geospace (ERG) project. In: Summers D et al (eds) Dynamics of the Earth's radiation belts and inner magnetosphere, Geophys. Monogr. Ser., vol 199. AGU, Washington, DC, pp 243-254. doi:10.1029/2012GM001304

Nunn D, Omura Y (2015) A computational and theoretical investigation of nonlinear wave-particle interactions in oblique whistlers. J Geophys Res Space Phys 120:2890-2911. doi:10.1002/2014JA020898

Omura Y, Nunn D (2011) Triggering process of whistler mode chorus emissions in the magnetosphere. J Geophys Res 116:A05205. doi:10.1029/2010JA016280

Omura Y, Katoh Y, Summers D (2008) Theory and simulation of the generation of whistler-mode chorus. J Geophys Res 113:A04223. doi:10.1029/200 7JA012622

Omura Y, Hikishima M, Katoh Y, Summers D, Yagitani S (2009) Nonlinear mechanisms of lower-band and upper-band VLF chorus emissions in the magnetosphere. J Geophys Res. doi:10.1029/2009JA014206

Omura Y, Nunn D, Summers D (2012) Generation process of whistler mode chorus emissions: current status of nonlinear wave growth theory. In: Summers D et al (eds) Dynamics of the Earth's radiation belts and inner magnetosphere, Geophys. Monogr. Ser., vol 199. AGU, Washington, DC, pp 243-254. doi:10.1029/2012GM001347 
Omura Y, Nakamura S, Kletzing CA, Summers D, Hikishima M (2015) Nonlinear wave growth theory of coherent hiss emissions in the plasmasphere. Geophys Res Space Phys 120:7642-7657. doi:10.1002/2015JA021520 Santolik O (2008) New results of investigations of whistler-mode chorus emissions. Nonlinear Process Geophys 15:621-630. doi:10.5194/ npg-15-621-2008

Santolik O, Gurnett DA, Pickett JS (2003) Spatio-temporal structure of stormtime chorus. J Geophys Res 108:1278. doi:10.1029/2002JA009791

Santolik O, Kletzing CA, Kurth WS, Hospodarsky GB, Bounds SR (2014) Fine structure of large-amplitude chorus wave packets. Geophys Res Lett 41:293-299. doi:10.1002/2013GL058889
Tang R, Summers D, Deng X (2014) Effects of cold electron number density variation on whistler-mode wave growth. Ann Geophys 32:889-898. doi:10.5194/angeo-32-889-2014

Thorne RM, Ni B, Tao X, Horne RB, Meredith NP (2010) Scattering by chorus waves as the dominant cause of diffuse auroral precipitation. Nature 467:943-946. doi:10.1038/nature09467

Yagitani S, Habagishi T, Omura Y (2014) Geotail observation of upper band and lower band chorus elements in the outer magnetosphere. J Geophys Res Space Phys 119:4694-4705. doi:10.1002/2013JA019678

\section{Submit your manuscript to a SpringerOpen ${ }^{\circ}$ journal and benefit from:}

- Convenient online submission

- Rigorous peer review

- Immediate publication on acceptance

- Open access: articles freely available online

- High visibility within the field

- Retaining the copyright to your article 